\section{Collapsed Objects in Clusters of Galaxies}

Application of the virial theorem to clusters of galaxies ${ }^{1-3}$ has shown that the masses of such elusters are unexpectedly large. For the Virgo Cluster ${ }^{4}$ a mass of $1 \times$ $10^{15} M_{\odot}$ is obtained from the virial theorem. This value is between one and two orders of magnitude larger than the combined mass of all of the known cluster galaxies. This suggests that most of the mass, which is required to stabilize the cluster, might be in the form of invisible collapsed objects.

Photographs of galaxies often show the tidal distortions that are produced by the gravitational interactions resulting from galaxy encounters. Similar distortions of galaxies would be produced by close encounters with very massive collapsed objects. Such events might be particularly frequent in rich clusters of galaxies.

To test this hypothesis the known members of the Virgo Cluster were inspected on the prints of the Palomar Sky Survey. Of the seventy-three known cluster members ${ }^{5}$, ten exhibit distortions which might be attributed to tidal interactions. Six of these ten galaxies have nearby companions that are almost certainly responsible for the observed distortions. The four isolated Virgo Cluster members that exhibit distortions are NGC 4192, NGC 4569, NGC 4866 and NGC 4958. It is, of course, quite possible that the distortions in some of these galaxies are due to the after effects of violent internal events rather than to tidal distortions.

These observations suggest that most distorted galaxies that are observed in the Virgo Cluster can be accounted for in terms of interactions with other galaxies. This in turn indicates that collapsed objects, with masses comparable with those of typical galaxies, are less frequent in the Virgo Cluster than are normal galaxies. This implies that collapsed objects with masses of, say, $5 \times 10^{10} M_{\odot}$ cannot account for the missing mass that would be required to stabilize the Virgo Cluster. The possibility that this mass is concentrated in a small number of exceedingly massive objects with masses larger than $10^{13} M_{\odot}$ cannot be excluded. Encounters between normal galaxies and a few such supermassive objects would be very rare. Also the observations cannot exclude the possibility that the missing mass is concentrated in a very large number of relatively small collapsed objects with masses less than $10^{8} M_{\odot}$. Presumably such relatively low-mass objects would not produce observable tidal distortions in normal galaxies. It is therefore concluded that rich clusters, such as the Virgo Cluster, probably do not contain large numbers of collapsed objects with masses in the range $10^{8} M_{\odot}$ to $10^{13} M_{\odot}$.

David Dunlap Observatory, StDney van DEN Bergh

University of Toronto,

Richmond Hill, Ontario,

Canada.

Received October 6, 1969.

' Zwicky, F., Helv. Phys. Acta, 6, 110 (1933).

2 Smith, S., Astrophys. J., 83, 23 (1936).

${ }^{3}$ Neyman, J., Page, T., and Scott, F., Astron. J., 66, 533 (1961).

‘ Van den Bergh, S., Astrophys. J., 131, 558 (1960),

'Humason, N. L., Mayall, N. U., and Sandage, A. R., Astron. J., 61, 97 (1956).

\section{Supernova of AD 1437}

KIANG $^{1}$ refers to the identification of the radio source CTB 35 as a supernova remnant and suggests a possible association with the Chinese supernova record of AD 1437. The only suggestion that CTB 35 is a supernova remnant was made by Braes and Hovenier ${ }^{2}$, who, incorrectly interpreting the radio data, deduced that CTB 35 had a non-thermal spectrum. In fact it has been shown from continuum observations ${ }^{3}$ and $\mathrm{Hl} 09 \alpha$ recombination line measurements ${ }^{4}$ that CTB 35 is a thermal complex well identified with emission nebulae in this direction. Tho suggestion that it is a supernova remnant is not justified.

The region within the Chinese asterism $W o e i^{1}$ is dominated by two sources: CTB 38, a thermal source also exhibiting recombination lines ${ }^{4}$, and CTB 37 , definitely a non-thermal double source ${ }^{3}$. The radio surface brightness of this latter source is comparable with that of the rem. nants of Tycho's Nova and of Kepler's Nova and it could be of the same age as these two remnants. It is therefore possible that CTB 37 is the remnant of the AD 1437 event.

Immediately outside and close to the second and third stars in Woei there is another non-thermal source, Kesteven No. 45 (ref. 5). This object is of low radio surface brightness, and if it is a supernova remnant it would be from a much earlier event than the events discussed by Kiang.

CSIRO Division of Radiophysics,

D. K. Mirine

Sydney,

Australia.

Received September 4, 1969.

${ }^{2}$ Kiang, T., Nature, 223, 599 (1969).

${ }^{2}$ Braes, L. E., and Hovenier, J. W., Bull. Astron. Insts. Netherlands, 18, 294 $(1966)$.

${ }^{3}$ Milne, D. K., and Hill, E. R., Austral. J. Phys., 22, 211 (1969).

' Wilson, T. L., thesis, M.I.T. (1969).

${ }^{5}$ Kesteven, M. J. L., Austral. J. Phys., 21, 369 (1968).

\section{Alleged Supernova of AD 1006}

FURTHER information obtained recently ${ }^{1}$ makes it likely that the identification of this object with $x$ Lupi is doubtful ${ }^{2}$ because the corrected declination for the epoch would most likely have been $-44^{\circ}$, too far south to have been seen from St Gallen.

Rockmount Hotel,

Cicely M. Botley

Tunbridge Wells,

Kent.

Received September 4, 1969.

${ }^{1}$ Goldstein, B. R., Astron. J., "70, 105 (1365).

${ }^{2}$ Botley, C. M., Nature, 222, 649 (1969).

\section{Linear Polarization of Pulsar PSR 0833-45 at $4.8 \mathrm{GHz}$}

OBSERVATIONS of the pulsar PSR $0833-45$ at $6 \mathrm{~cm}$ wavelength have shown a double structure for the pulse, a drop in the polarization from 100 per cent to 50 per cent near the pulse maximum, and a non-linear variation of polarization angle across the pulse. These observations are interpreted in terms of an oblique rotator model for the pulsar.

PSR 0833-45 has been observed at frequencies of $1,420 \mathrm{MHz}, 1,720 \mathrm{MHz}$ and $2,700 \mathrm{MHz}$ by Radhakrishnan et $a l .{ }^{1}$ and by Radhakrishnan and Cooke ${ }^{2}$. On the basis of the polarization features it was concluded that the pulses were due to highly beamed radiation from the vicinity of the magnetic poles of a neutron star that was rotating rapidly with a period of $89 \mathrm{~ms}$. The observations described here were to investigate the pulsar characteristics at a frequency of $4,830 \mathrm{MHz}$.

The observations were carried out on May 19, 1969, with the Parkes 210 foot reflector using a $6 \mathrm{~cm}$ parametric receiver on loan from the National Radio 